\title{
Polygalacturonase and Polygalacturonase Inhibitor Protein: Gene Isolation and Transcription in Glycine max - Heterodera glycines Interactions
}

\author{
Ramamurthy Mahalingam, ${ }^{1}$ Gejiao Wang, ${ }^{2}$ and Halina T. Knap ${ }^{3}$ \\ ${ }^{1}$ Genetics Program, Clemson University, Clemson, SC 29634, U.S.A.; ${ }^{2}$ Department of Crop \& Soil Environ- \\ mental Science, Clemson University, Clemson, SC 29634, U.S.A.; ${ }^{3}$ Department of Crop \& Soil Environ- \\ mental Science, Department of Biological Sciences, Clemson University, Clemson, SC 29634, U.S.A. \\ Accepted 20 February 1999.
}

\begin{abstract}
The cell wall acts as the first line of defense during pathogen invasion. Polygalacturonases (PGs) are a class of cellwall-modifying enzymes with precise temporal and organspecific expression. A 350-bp fragment with high homology to $P G$ s was identified by differential display (DD) analysis of soybean cyst nematode $(\mathrm{SCN})$ race 3 resistant PI 437654 and susceptible cultivar Essex. The fragment was strongly expressed in Essex, 2 days after inoculation (DAI). Complete coding sequences of two $P G$ cDNAs, $P G 1$ and $P G 2$, were isolated by $3^{\prime}$ and $5^{\prime}$ rapid amplification of cDNA ends polymerase chain reaction (RACE PCR). PI 437654 and Essex had identical $P G 1$ and $P G 2$ sequences. A transversion from A to $\mathrm{C}$ created a $P$ stI restriction site in the $P G 2$ cDNA that was used to distinguish the two $P G$ cDNAs by cleaved amplified polymorphic sequence (CAPS) analysis. A cDNA encoding a polygalacturonaseinhibitor protein (PGIP) that is $89 \%$ identical to the Phaseolus vulgaris PGIP was isolated from soybean roots by reverse transcription (RT)-PCR. Steady-state levels of $P G$ and $P G I P$ were investigated by RNA gel blot analysis in roots 1 to 5 DAI and in hypocotyls and leaves. Differences in the constitutive levels of $P G$ mRNAs were observed in roots of different soybean genotypes. Steadystate levels of $P G$ mRNAs were enhanced during compatible interactions with $\mathrm{SCN}$ and reduced in incompatible interactions and in mechanically wounded roots. Enhanced PGIP transcription was observed in response to mechanical wounding in both PI 437654 and Essex, but only in compatible interactions with $\mathrm{SCN}$, suggesting uncoupling of PGIP functions in developmental and stress cues. Constitutive expression in incompatible interactions shows PGIP is not a factor in SCN resistance. Thus, the up-regulation of endogenous $P G$ transcription in soybean roots early after SCN infection could facilitate successful parasitism by SCN.
\end{abstract}

Corresponding author: Halina T. Knap; E-mail: hskrpsk@clemson.edu

Current address of Ramamurthy Mahalingam: 211 Wartik Laboratory, Biotechnology Institute, Pennsylvania State University, University Park 16802, U.S.A.

Nucleotide and/or amino acid sequence data are to be found at the GenBank data base as accession nos. AF128266, AF128267, and AF130974.
Additional keywords: oligogalacturonides, open reading frame, syncytia, systemic, transduction.

Pectins, also referred to as matrix polysaccharides, are a major component of the plant cell wall and glue neighboring cells together. Pectin metabolism is crucial to many developmental processes. Plant cells undergo drastic changes in shape and developmentally regulated cell separation, during which the pectin network is systematically disassembled. A wide range of enzymes, including, exo- and endo-polygalacturonases (PGs), pectate lyase, pectin methyl esterase, and betagalactosidase, are involved in pectin modifications (Hadfield and Bennett 1998).

In many plant developmental processes, particularly those that require cell separation, PGs cleave the $\propto(1-4)$ linkages of D-galacturonic acid, which forms the backbone of pectins. Several $P G$ s have been cloned in various plant species and show time- and organ-specific expression (Brown and Crouch 1990; DellaPenna et al. 1986; Slater et al. 1985; Taylor et al. 1990; Tebutt et al. 1994; Wing et al. 1989).

During pathogenesis, cell walls act as the first line of defense that phytopathogens encounter to colonize the plant tissue and obtain nutritional requirements. Plant-pathogenic bacteria and fungi have evolved a battery of pectic enzymes, primarily endo-PGs, to hydrolyze the plant cell walls during the early stages of pathogenesis (reviewed in Hahn et al. 1989). In the more evolved types of parasitism, such as the one between soybean cyst nematode ( $\mathrm{SCN}$ ) and soybean, the endoparasite has developed sophisticated means of exploiting the host plant. A long and stout stylet aids the nematode in penetrating several cell layers in search of food. The sessile nature of developing juveniles (J3, J4 stages) and adult females of SCN helps to limit necrotic plant reactions detrimental to nematode development. For the successful completion of the life cycle, SCN must initiate localized reorganization of the host cell's morphology, resulting in the formation of specialized feeding sites or syncytia (Jones 1981). Dissolution of walls of the cells surrounding the initial feeding site leads to the formation of multi-cellular syncytia. The cell wall dissolution is an enzymatic process, as observed in infected roots of susceptible cultivar Essex (Mahalingam and Skorupska 1996). In both SCN-compatible and SCNincompatible soybeans, syncytia are initiated as early as 2 
days after inoculation (DAI; Mahalingam and Skorupska 1996). We report the changes in the steady-state levels of soybean $P G$ transcripts that may play a role during early stages of soybean-SCN interactions.

All dicotyledonous plants have a cell-wall-localized polygalacturonase-inhibitor protein (PGIP). PGIPs are specific, high-affinity receptors for fungal endo-PGs (Hahn et al. 1989). Formation of the PG-PGIP complex in vitro results in an alteration of the balance between release of elicitor-active oligogalacturonides and depolymerization of oligogalacturonides into inactive molecules, favoring the accumulation of elicitor-active molecules (Cervone et al. 1989). The plant resistance genes that participate in gene-for-gene resistance$C f-2, C f-9$ of tomato, and $X a 21$ of rice-show high homology to the PGIPs (Jones and Jones 1997). The receptor-like, leucine-rich, modular structure of the PGIP provides for the function of PGIP as a component of the cell-surface signaling system (De Lorenzo et al. 1994). We observed an induction of $P G I P$ when there was an induction of $P G$ in response to pathogen but not wounding. This induction was specific to compatible interactions, and regulatory mechanisms have yet to be identified. We tested PGIP expression to investigate if a general resistance gene like $P G I P$ may be important in soybean response to $\mathrm{SCN}$. The transcription of PGIP during the soybean-SCN interactions excludes PGIP as a factor in resistance response.

\section{RESULTS}

\section{Two $P G$ coding sequences were identified} from soybean root cDNAs.

A 350-bp differential display (DD) fragment was amplified from cDNA generated from Essex roots infected with SCN race 3, 2 DAI (Fig. 1). This amplicon was reproducibly obtained with independent RNA isolations and at two concentrations of the template (not shown). The fragment was not observed in the root cDNAs of PI 437654. The sequence of the fragment was $343 \mathrm{bp}$ long and contained the decamer sequence at both $5^{\prime}$ and $3^{\prime}$ ends. This fragment had an open reading frame (ORF) of 112 amino acids (aa) that showed homology to PGs from several plant, bacterial, and fungal species. The highest homology was with Arabidopsis PG (74\% identity; BLAST score, $377 ; P=1.2 \mathrm{e}-46$ ).

To obtain the full-length sequence of the soybean $P G$ cDNA, the DD fragment was subjected to $5^{\prime}$ and $3^{\prime}$ rapid amplification of cDNA ends polymerase chain reaction (RACE PCR). Sequencing of more than a dozen clones generated by RACE PCR from PI 437654 and Essex indicated that there were two $P G$ coding sequences (named $P G 1$ and $P G 2$; Fig. 2). Both $P G 1$ and $P G 2$ cDNA sequences were identical in Essex and PI 437654. PG1 cDNA predicted a single ORF encoding 443 aa (Fig. 3) with the start codon at nucleotide 73 and the stop codon at nucleotide 1402. PG2 cDNA sequence revealed an ORF of 439 aa (Fig. 3) with the start codon at nucleotide 85 and the stop codon at nucleotide 1402. Apart from PG2 being 4 aa smaller than PG1, there were 23 aa substitutions between PG1 and PG2 sequences. The molecular masses of the deduced peptide of PG1 and PG2 were estimated as 47.3 and $47.1 \mathrm{kDa}$, respectively. The diagnostically conserved sequence, CGPGHGISIGSL, identified in all the PGs cloned to date (Petersen et al. 1996; Hadfield et al. 1998), was pres- ent in both PG1 and PG2, between positions 269 and 280 in PG1 (Fig. 3). $P G 1$ and $P G 2$ had overall $92 \%$ nucleotide identity: $\left(94 \%\right.$ in the ORF, $82 \%$ in the $5^{\prime}$ untranslated region [UTR] and $86 \%$ in the $3^{\prime}$ UTR). A transversion from A to C created a PstI restriction site in the $P G 2$ sequence (Fig. 2). In cleaved amplified polymorphic sequence (CAPS) analysis, the 1,400-bp amplification product from the $P G 1$-specific primers, (namely PG1-1 as the $5^{\prime}$ primer and PG1-3 as the $3^{\prime}$ primer) lacked the PstI restriction site and hence remained uncut after digestion (Fig. 4). Amplification of root cDNA with $P G 2$-specific primers, namely PG2-1 as the $5^{\prime}$ primer and PG2-3 as $3^{\prime}$ primer, followed by restriction digestion with PstI generated 1,160- and 240-bp fragments. Nonspecific combinations of primers (e.g., PG1-1/PG2-3 or PG2-1/PG1-3) failed to produce any amplification products (Fig. 4).

$P G 1$ and $P G 2$ cDNAs were used as probes on DNA gel blots of PI 437654 and Essex digested with five different restriction enzymes, namely, DraI, EcoRI, EcoRV, HindIII, and TaqI. Both PG1 and PG2 cDNAs hybridized to 4 to 10 genomic fragments, and the hybridization patterns were identical. Several weakly hybridizing bands were observed under high stringency washing conditions, indicating the presence of other $P G$-related sequences in soybean genome.

\section{A novel $P G I P$ sequence from soybean roots.}

PCR amplification from genomic DNA and root cDNAs of PI 437654 and Essex with the primers for soybean PGIP according to Favaron et al. (1994) revealed an expected 950-bp fragment (Fig. 5). Sequences of PGIP clones from roots of PI

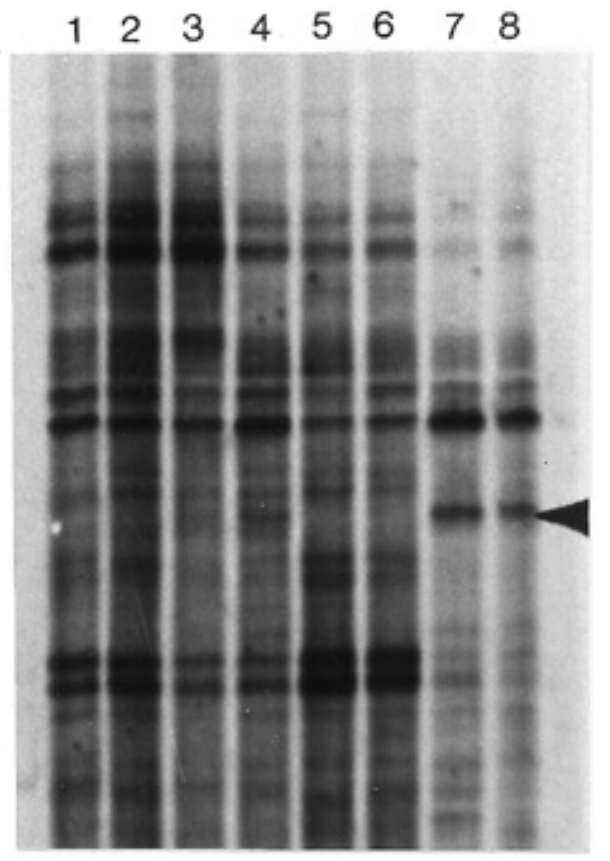

Fig. 1. Differential display of cDNAs generated from $\mathrm{T}_{17}-\mathrm{C}$ anchor primer in combination with the decamer primer 5'-TCCGATGCTG-3' and separated on a $6 \%$ denaturing polyacrylamide gel. Only a portion of the autoradiogram with the differentially expressed polygalacturonase $(P G)$ fragment (indicated by an arrow) is shown. Lanes 1 and 2: noninfected PI 437654 roots; lanes 3 and 4: PI 437654 roots 2 days after inoculation with race 3 soybean cyst nematode; lanes 5 and 6: noninfected roots of Essex; lanes 7 and 8: Essex roots 2 days after inoculation with race 3 soybean cyst nematode. 
437654 and Essex were 100\% identical and contained perfectly matching sequences for the $5^{\prime}$ and $3^{\prime}$ primers. The genomic clone of PGIP published by Favaron et al. (1994) was $77 \%$ identical at nucleotide level to the PGIP sequence we identified in roots (Fig. 6). At amino acid level, the sequence of PGIP from roots (Gm PGIP) was $88 \%$ identical to that of the common bean, Phaseolus vulgaris, (Pv PGIP) sequence while the PGIP reported by Favaron et al. (Gmc PGIP; Favaron et al. 1994) was 67\% identical to the Pv PGIP (Fig. 7).

Southern blot analysis with PGIP cDNA showed one strongly hybridizing fragment after digestion with EcoRI and two strongly hybridizing fragments after HindIII digestion, apart from several other weakly hybridizing fragments. Only one HindIII site and no EcoRI restriction sites are present in this PGIP sequence.

\section{$P G$ transcription is enhanced in compatible roots early after SCN infection.}

RNA blot hybridization with the full-length $P G$ cDNA required long exposure of up to 7 days, indicating low levels of $P G$ transcripts in roots. We could not differentiate between $P G 1$ and $P G 2$ by RNA blot analysis. The levels of $P G$ transcripts differed between PI 437654 and Essex in the noninfected roots. In the susceptible cultivar Essex, the steady-state levels of $P G$ in the noninfected roots were higher while in the resistant PI $437654 P G$ was hardly detectable, confirming ex-

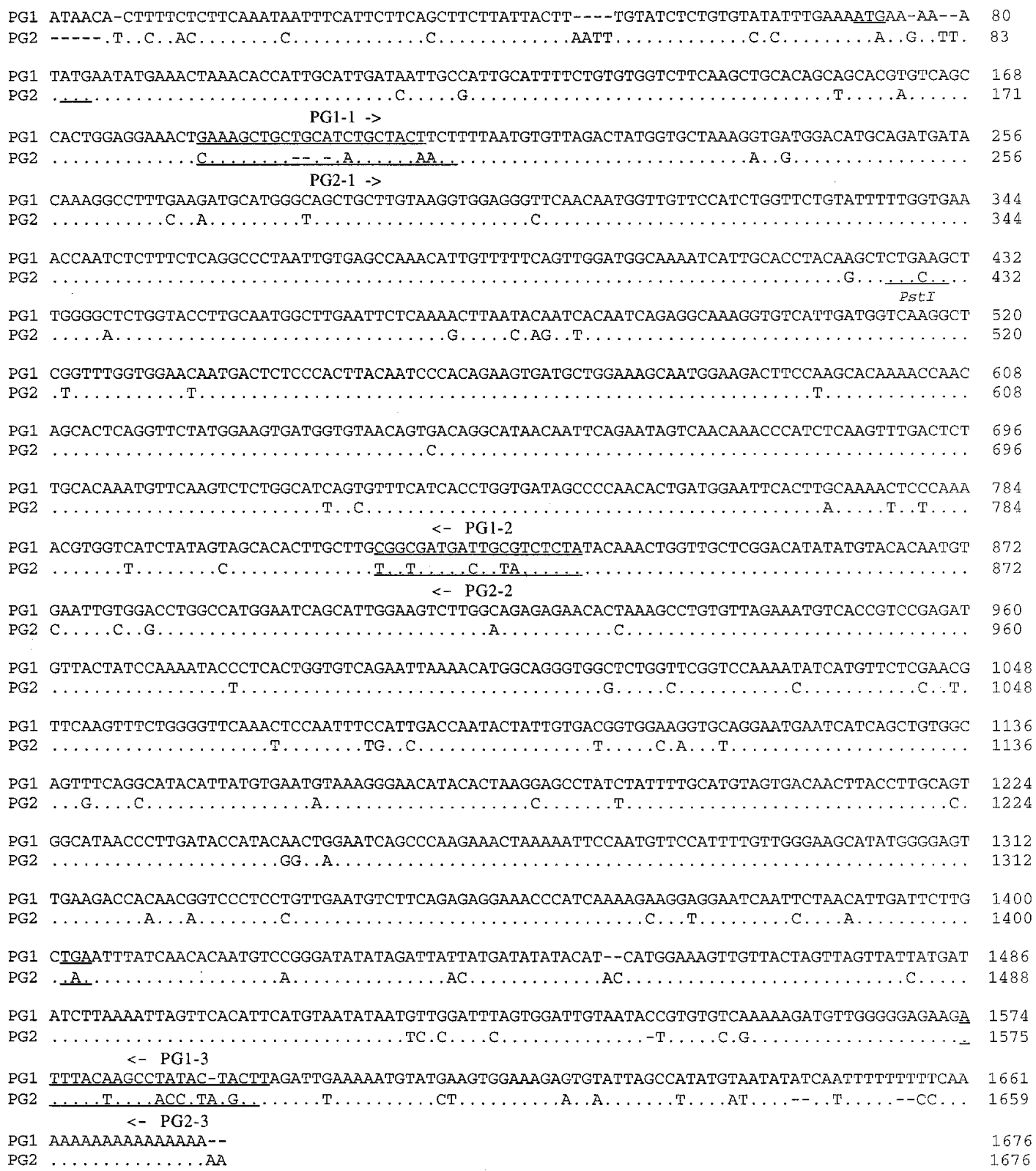

Fig. 2. Nucleotide sequences of the full-length cDNAs of $P G 1$ and $P G 2$. The gene-specific primers used for the cleaved amplified polymorphic sequence (CAPS) analysis are underlined. Identical nucleotides between $P G 1$ and $P G 2$ sequences are indicated by dots, and deletions by dashes in the $P G 2$ sequence. The first start codon and the stop codon are underlined. The PstI recognition site present only in the $P G 2$ sequence is indicated. Primers used in the CAPS analysis are shown: right arrows next to the primer name indicate forward or $5^{\prime}$ primer; left arrows indicate reverse or $3^{\prime}$ primer. 
pression in DD analysis (Fig. 8A). In the SCN-susceptible cultivars Dillon, Brim, Perrin, Lee, and Hutcheson, hybridization to $P G$ transcript was distinct (data not shown). No hybridization with $P G$ cDNAs was detectable in PI 438/489B (resistant to all races of SCN tested; Diers et al. 1997) and PI 209332 (resistant to races 3 and 14, susceptible to race 1).

After infection with $\mathrm{SCN}$ race $3, P G$ transcripts were induced in the infected roots of Essex as early as 1 DAI, reaching the highest levels 3 DAI. $P G$ expression returned to the same level as in the noninfected roots by 5 DAI (Fig. 8A). In infected roots of PI 437654, a faint signal was detected 1, 1.5, 2 , and 3 DAI. There was no detectable signal 5 DAI. In mechanically wounded roots, the $P G$ transcripts were totally down-regulated (Fig. 8A).

In leaf RNA of PI 437654 and Essex, there was no detectable signal with $P G$ cDNAs (Fig. 8B). In hypocotyls, $P G$ transcript levels slightly increased after infection in Essex, while in PI 437654 they were down-regulated (Fig. 8B).

In compatible host-race combinations, Peking and PI 88788 infected with SCN race 14, transcripts were detected with the $P G$ cDNAs in roots (Fig. 8C). In Essex roots infected with race 14 , the steady-state levels of $P G$ cDNAs were the highest. In incompatible host-race combinations, namely, Peking and PI 88788 infected with race 3, $P G$ transcripts were undetectable (Fig. 8C). Interestingly, in PI 437654, which is resistant to race 14 , a clear signal was detected after hybridization with the $P G$ cDNAs (Fig. 8C).

PGIP transcription is induced in response to stress.

In contrast to weak hybridization signals with $P G$ cDNAs, $P G I P$ was expressed in all the tissues tested (Fig. 8A and B). The RNA blot analysis of roots revealed a 1,400-nucleotide transcript when hybridized with PGIP cDNA (Fig. 8A). After SCN infection, PGIP expression was strong 2 DAI, and 5 DAI in Essex (Fig. 8A). In PI 437654, no differential expres- sion was observed in response to $\mathrm{SCN}$ infection. In the mechanically wounded roots of both the genotypes, there was clear induction of PGIP expression (Fig. 8A).

In the leaf RNA, there was one strong hybridizing band, about $1.4 \mathrm{~kb}$, that after infection showed a slightly elevated expression in Essex but not in PI 437654 (Fig. 8B). In hypocotyl RNA, two transcripts were detected (Fig. 8B). The smaller transcript $(1.4 \mathrm{~kb})$ was strongly expressed in infected

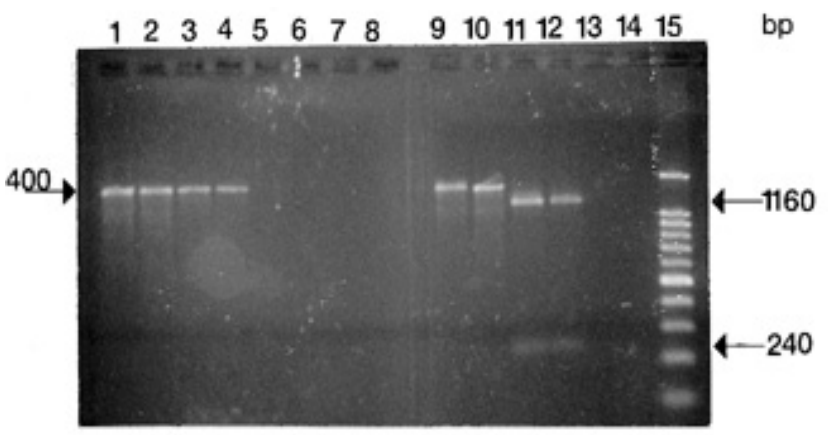

Fig. 4. Cleaved amplified polymorphic sequence (CAPS) analysis of $P G 1$ and $P G 2$ by reverse transcription-polymerase chain reaction (RTPCR) followed by digestion with PstI. Amplifications were carried out with the gene-specific primers shown in Figure 2. RT-PCR was conducted for 30 cycles at $94^{\circ} \mathrm{C}$ for $40 \mathrm{~s}, 59^{\circ} \mathrm{C}$ for $40 \mathrm{~s}$, and $72^{\circ} \mathrm{C}$ for $2 \mathrm{~min}$. Two fragments, 1,160 and $240 \mathrm{bp}$, were observed after digestion of the $P G 2$ amplification product with the PstI enzyme. Lane 1: PG1-1/PG1-3 (Essex); lane 2: PG1-1/PG1-3 (PI 437654); lane 3: PG2-1/PG2-3 (Essex); lane 4: PG2-1/PG2-3 (PI 437654); lane 5: PG1-1/PG2-3 (Essex); lane 6: PG1-1/PG2-3 (PI 437654); lane 7: PG2-1/PG1-3 (Essex); lane 8: PG2-1/PG1-3 (PI 437654); lane 9: PG1-1/PG1-3 (Essex)- PstI; lane 10: PG1-1/PG1-3 (PI 437654)-PstI; lane 11: PG21/PG2-3 (Essex)-PstI; lane 12: PG2-1/PG2-3 (PI 437654)-PstI. Negative controls for cDNA synthesis without reverse transcriptase, lane 13: PI 437654; and lane 14: Essex. Lane 15: 100-bp DNA ladder (Promega, Madison, WI).

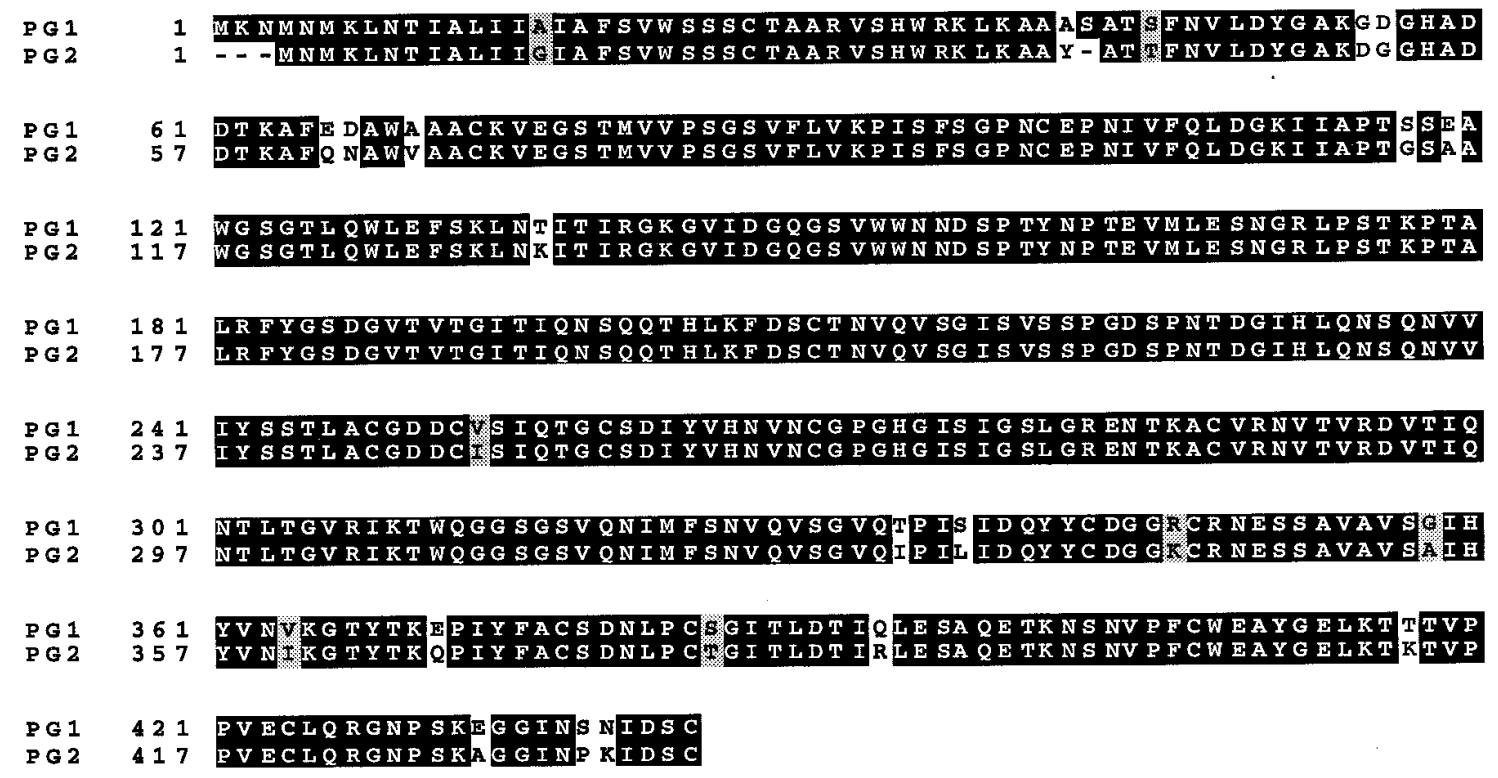

Fig. 3. Deduced amino acid sequence of $P G 1$ and $P G 2$ full-length cDNAs. Deletions are indicated by dashes in the $P G 2$ sequence. Sequence alignments were performed with the CLUSTAL W multiple sequence alignment program (version 1.7, 1997; found on-line from the Human Genome Center, Baylor College of Medicine) and viewed with BOXSHADE (found on-line from ISREC [the Swiss Institute for Experimental Cancer Research]). Regions of identity (black) and similarity (shaded) are indicated. Dissimilar amino acids are in white boxes. 
PI 437654, while in Essex no differences were detected in the amount of smaller transcript after infection. The larger transcript $(2.2 \mathrm{~kb})$ showed reduced expression after infection in both genotypes.

In compatible host-race interactions, i.e., Essex, Peking, and PI 88788 infected with SCN race 14, there was a stronger expression of $P G I P$ than in the incompatible interaction in

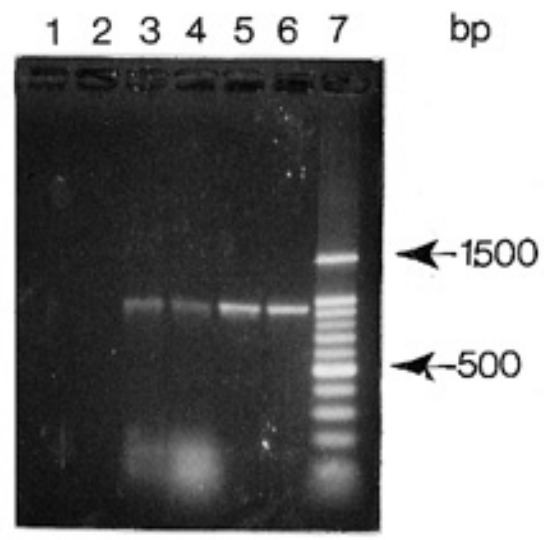

Fig. 5. Polygalacturonase-inhibitor protein (PGIP) gene amplification from genomic DNA and cDNAs with the primers described in Favaron et al. (1994). The amplification products were resolved on a $1.4 \%$ agarose gel. Amplification from genomic DNA of PI 437654 with the following. Lane 1: $P G I P-5^{\prime}$ primer alone; lane 2: $P G I P-3^{\prime}$ primer alone; lane 3: PGIP 5' and $3^{\prime}$ primers; lane 4: Amplification from Essex DNA with PGIP 5' and 3' primers; lanes 5 and 6: Amplification of cDNA with PGIP 5' and 3' primers. Lane 5: PI 437654; lane 6: Essex; lane 7: 100bp DNA ladder (Promega, Madison, WI).
Peking and PI 88788 infected with race 3 (Fig. 8C). There were no detectable differences in the abundance of PGIP transcript levels in PI 437654 infected with races 3 or 14 (Fig. 8C).

\section{DISCUSSION}

\section{$P G$ transcription differs in soybean-SCN interactions.}

All the $P G$ s cloned to date in plants are associated with developmental processes (Hadfield and Bennett 1998). Recently, a Medicago sativa $P G$ gene was shown to be involved in the early stages of the Rhizobium meliloti-legume symbiosis (Munoz et al. 1998). For the first time, we report a plant $P G$ that is differentially expressed in a plant-pathogen interaction. Detection of $P G$ expression in roots of soybean required prolonged autoradiography (5 to 7 days) of RNA blot analysis, indicating low levels of $P G$ transcripts. In this context, detection of a differentially expressed fragment of low abundance level demonstrates sensitivity of the DD methodology.

Extensive sequencing of the $5^{\prime}$ and $3^{\prime}$ RACE products (27 clones) aided in the identification of two $P G$ cDNAs from soybean roots. Existence of duplicate genes in soybean is not surprising because of the polyploidization event in its ancestry. Even when the 3' UTR of the cDNAs was used for the Southern blot analysis, restriction fragment length polymorphism (RFLP) patterns with $P G 1$ and $P G 2$ cDNAs were identical (not shown), suggesting that the duplication event may have occurred recently during evolution. The two $P G$ cDNAs were $92 \%$ identical at the nucleotide level and could be distinguished by CAPS analysis with PstI. Although RNA blot analysis clearly showed only one band, it is possible that the

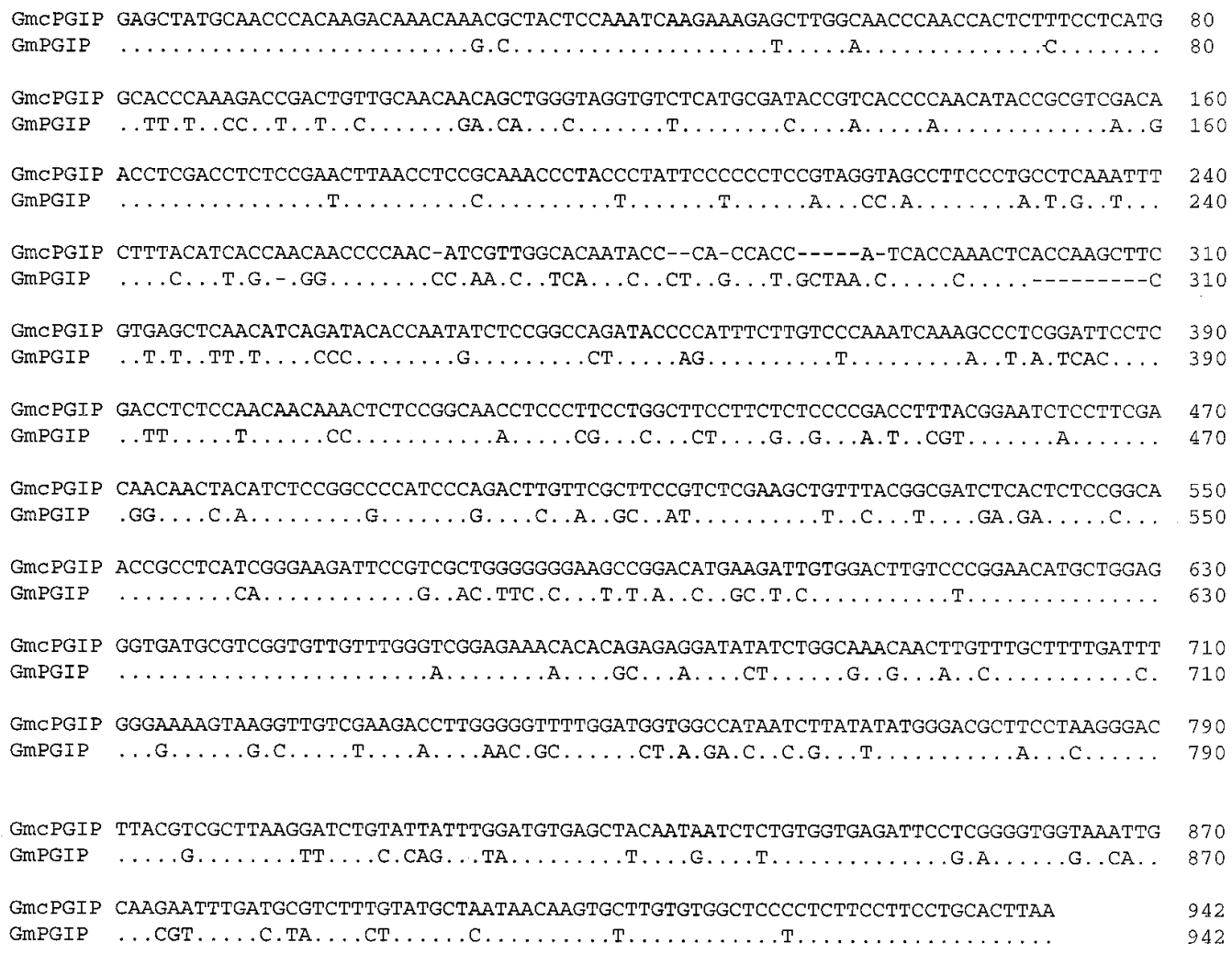

Fig. 6. Comparison of the nucleotide sequence of GmPGIP (this research) and GmcPGIP (Favaron et al. 1994). Identical nucleotides between GmPGIP and GmcPGIP are indicated by dots. 
two $P G$ cDNAs may produce similar sized transcripts that cannot be resolved by RNA blot analysis. Using the 3' UTR of the cDNA clones (approximately $250 \mathrm{bp}$ ), we could not differentiate between steady-state levels of $P G 1$ and $P G 2$. However, CAPS analysis showed that both $P G 1$ and $P G 2$ are expressed in the roots of both PI 437654 and Essex. In tomato, there is only one $P G$ gene per haploid genome that is expressed exclusively during fruit ripening (Bird et al. 1988). The $P G$ gene, detected in flower abscission zones in tomato, was only $43 \%$ identical to the fruit $P G$, and it failed to crosshybridize (Kalatizis et al. 1995). Similarly, the $P G$ s in soybean leaves may be significantly divergent from the root $P G$ s, since no signal was detectable with the root $P G$ in RNA blot analysis. Both the identified $P G$ cDNAs were expressed in roots and hypocotyls and produced transcripts of the same size. Although constitutively expressed, $P G$ transcription was genotype dependent, as shown by different levels of $P G$ transcripts in the 14 soybean genotypes tested. Furthermore, $P G$ transcription was associated with host reaction to SCN. The levels of $P G$ cDNAs were down-regulated in the incompatible interactions with race 3 . The enhanced expression of $P G$ cDNAs in compatible hosts for SCN race 14 (Peking, PI 88788) and greatly elevated levels of $P G$ s in Essex infected with race 14 , compared with infection with race 3 , suggest that the nematode genotype may play a role in $P G$ transcription.

Cellular rearrangements during the formation of the feeding site by sedentary parasitic nematodes in general and SCN in particular utilize portions of the normal plant developmental program (Bird 1996; Mahalingam and Skorupska 1996). Grundler et al. (1994) reported that the procambial cells recruited in syncytium formation by Heterodera schachtii in Arabidopsis were xylem initials and this was responsible for the observed suppression of xylem formation. However, Bird (1996) suggested that, rather than suppressing xylem formation, syncytium formation depletes the supply of xylem precursors. Bird (1996) also suggested that precocious expression of developmentally programmed cell wall dissolution, char- acteristic of final stages of xylem differentiation, may be responsible for syncytium formation. The identification of plant PG cDNAs that catalyze the hydrolysis of cell wall $\alpha(1-4)$ linkages of D-galacturonans, and are up-regulated as early as 1 DAI, corroborates Bird's (1996) observations and supports the hypothesis that developmentally regulated genes may be involved in syncytium formation (Mahalingam and Skorupska 1996). There was no cross-hybridization of $P G$ cDNA to SCN genomic DNA, indicating that the $P G$ is of soybean origin (data not shown).

The total down-regulation of $P G$ transcripts in hypocotyls of PI 437654 after SCN infection indicates that the signal transduction machinery responsible for their suppression is very strong. We speculate that PGs are not efficient in cell wall breakdown in PI 437654 because of possible trans-acting factors such as the inhibitors of PGs, or the cis-acting nematode responsive sequences (if any) essential for increased expression are not functional. Further, the higher abundance of $P G$ transcripts in hosts susceptible to at least one race of SCN strongly suggests that steady-state levels of $P G$ may be one of the key factors for establishment of syncytium. The differences in the observed patterns of expression between compatible Essex and incompatible PI 437654 probably reside in the upstream promoter sequences of the $P G$ genes since there are no differences between the two genotypes in the ORFs of $P G 1$ and $P G 2$. Mechanically wounded roots were included in this analysis to verify whether the induction of $P G$ expression was a general response to stress. The down-regulation of $P G$ transcription in the wounded roots further exemplified that enhanced $P G$ transcription in response to $\mathrm{SCN}$ infection is specific and may provide cues for manipulating response to $\mathrm{SCN}$.

\section{PGIP transcription differs in soybean-SCN interactions.}

We isolated a PGIP sequence from the root cDNA of PI 437654 and Essex that shows $72 \%$ amino acid homology to the reported PGIP from genomic DNA of soybean cultivar Canton (Favaron et al. 1994). To confirm that this was not due

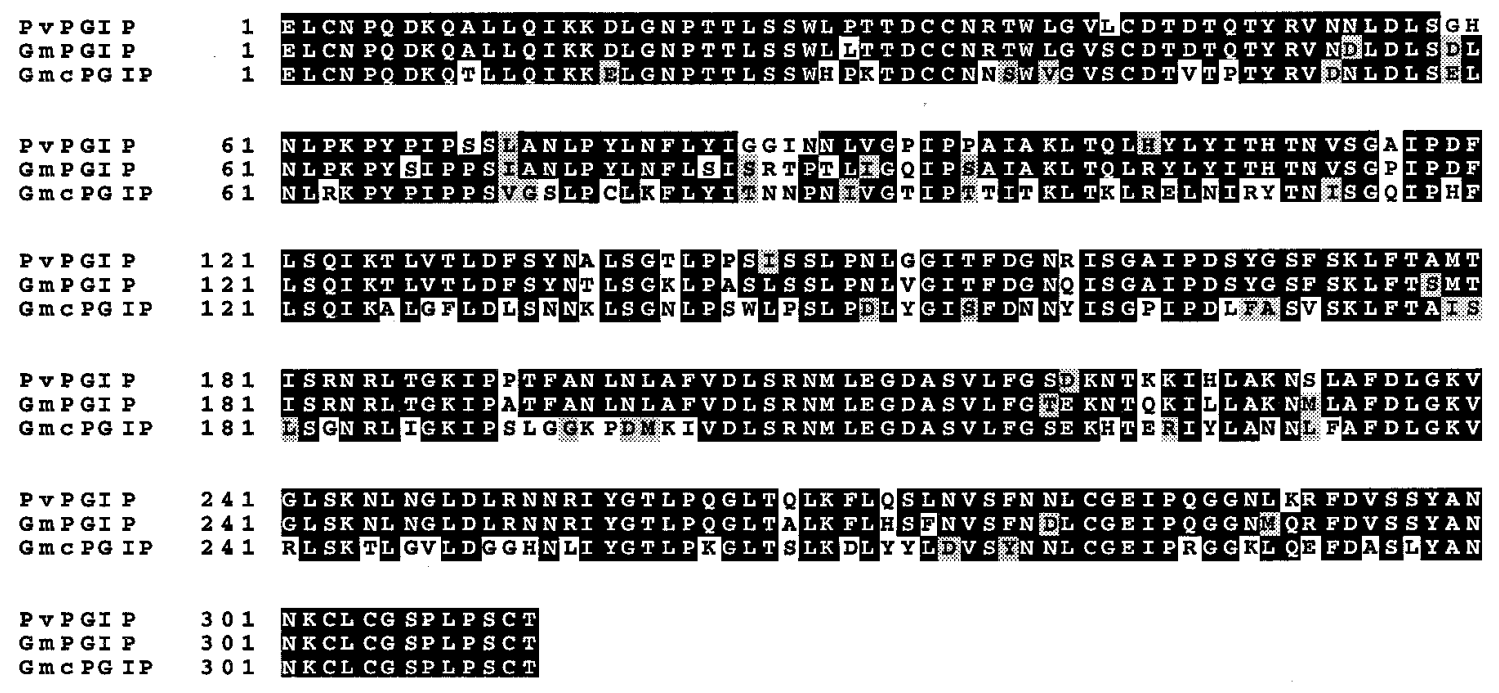

Fig. 7. Comparison of the deduced amino acid sequences of polygalacturonase-inhibitor proteins (PGIPs). Shown are the sequences of Phaseolus vulgaris PGIP (PvPGIP), Glycine max PGIP from roots (GmPGIP, this research), and GmcPGIP (Favaron et al. 1994). Sequence alignments were performed with the CLUSTAL W multiple sequence alignment program (version 1.7, 1997; found on-line from the Human Genome Center, Baylor College of Medicine) and viewed with BOXSHADE (found on-line from ISREC [the Swiss Institute for Experimental Cancer Research]). Regions of identity (black) and similarity (shaded) are indicated. Dissimilar amino acids are in white boxes. 
to sequencing errors, several additional clones from Essex and PI 437654 were sequenced. All sequenced clones (four from PI 437654 and four from Essex) were 100\% identical but showed low homology to the earlier reported PGIP. Favaron et al. (1994) sequenced only one genomic clone of PGIP and confirmed its sequence by direct sequencing of the PCRamplified product. Furthermore, the sequence of the PGIP we obtained is $88 \%$ identical to the common bean (Phaseolus vulgaris) sequence and hence we claim that our sequence represents PGIP of soybean. The sizes of the fragments generated from the cDNAs and genomic DNA were identical, indicating that PGIP is an intronless gene (Fig. 5). The presence of other weakly hybridizing fragments in Southern blot analysis indicates that there are other $P G I P$-like sequences in the soybean genome.

\section{A}

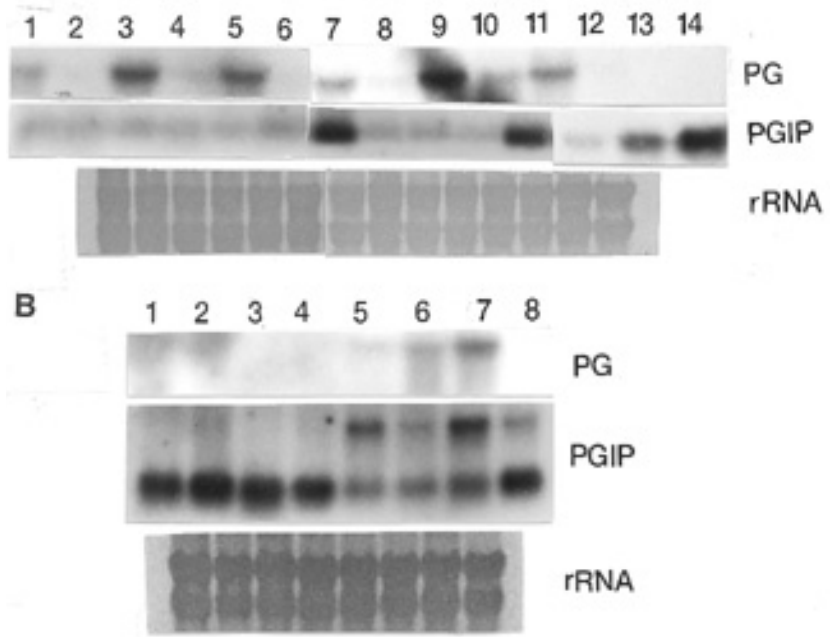

C

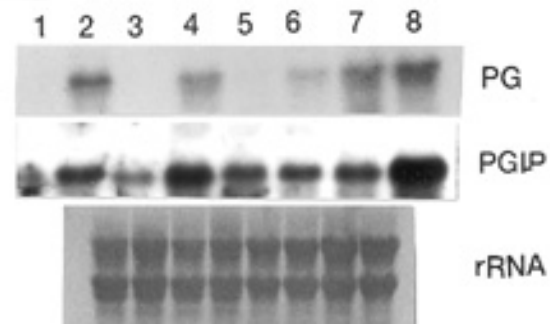

Fig. 8. RNA blot analysis with the polygalacturonase $(P G)$ and polygalacturonase-inhibitor protein $(P G I P)$ cDNA clones. Ten micrograms of total RNA was separated on a $1.2 \%$ formaldehyde-agarose gel. A, Temporal expression analysis of $P G$ and $P G I P$ cDNAs in soybean-soybean cyst nematode (SCN) interactions. Lane 1: noninfected roots of Essex; lane 2: non-infected roots of PI 437654; lane 3: Essex, lane 4: PI 437654, 1 day after inoculation (DAI); lane 5: Essex; lane 6: PI 437654 1.5 DAI; lane 7: Essex; lane 8: PI 437654, 2 DAI; lane 9: Essex; lane 10: PI 437654, 3 DAI; lane 11: Essex; lane 12: PI 437654, 5 DAI; lane 13: wounded roots of Essex; and lane 14: wounded roots of PI 437654. The third panel shows the methylene blue staining of the rRNA to demonstrate loading of RNA. B, Systemic expression analysis of $P G$ and $P G I P$ cDNAs in soybean-SCN interactions. Leaf: lane 1: Noninfected Essex; lane 2: Essex, 2 DAI; lane 3: noninfected PI 437654; lane 4: PI 437654, 2 DAI; Hypocotyls: lane 5: noninfected Essex; lane 6: Essex, 2 DAI; lane 7: noninfected PI 437654; and lane 8: PI 437654, 2 DAI. C, Race-specific responses of $P G$ and $P G I P$ cDNAs in SCN resistant and susceptible soybeans. Peking: lane 1: race 3; lane 2: race 14; PI88788: lane 3: race 3; lane 4: race 14; PI 437654: lane 5: race 3; lane 6: race 14; Essex: lane 7: race 3; and lane 8: race 14 .
PGIPs have been reported in all the plant tissues, including the floral organs. We observed strong PGIP expression in roots, leaves, and hypocotyls. Based on their ubiquitous expression, PGIPs have been regarded as being important for general resistance to biotic and abiotic stresses and also in cell wall repair mechanisms. PGIPs effectively inhibit fungal PGs (Desiderio et al. 1997; Stotz et al. 1993). In vitro, fungal endopolygalacturonases convert polygalacturonic acid of pectins to inactive mono-, di-, and even trigalacturonic acids (English et al. 1972). It is well established that cell wall fragments or oligogalacturonides are active elicitors in the induction of plant defense responses (Nothangel et al. 1983; WalkerSimmons et al. 1984; Cervone et al. 1989). Especially linear $\propto-(1 \rightarrow 4)-$ linked oligogalacturonides of chain lengths between 10 and 13 are strong inducers of phytoalexins and lignification; shorter oligomers have little or no elicitor activity (Hahn et al. 1981).

$P G I P$ is a classical resistance gene. If PGIP were a typical incompatibility factor in the soybean-SCN interaction, then its expression should be higher in the incompatible interactions than in the compatible interactions. However, we observed the opposite phenomenon in the soybean-SCN interactions, indicating that PGIP does not behave as a resistance factor. We speculate that $P G$ and $P G I P$ expression during normal plant development may be under mutual control at a transcriptional level. In such an instance, if the constitutive level of $P G$ expression is undisturbed then the constitutive levels of $P G I P$ remain unaltered. If the levels of $P G$ transcripts increase (e.g., after SCN infection), then a default mechanism would trigger levels of $P G I P$ as seen in the compatible interactions. We observed an induction of $P G I P$ only when there was an induction of $P G$ in response to pathogen but not mechanical wounding. This observed induction was not genotype specific; on the contrary, it was interaction specific. In all the compatible host-race combinations tested, we observed increases in $P G$ and $P G I P$ transcripts. Increased level of $P G$ s implies more wall breakage, and this would eventually lead to the formation of elicitor-active oligogalacturonides that is detrimental to a compatible response. If PGIP would be brought into the system, it may bring equilibrium favoring the suppression of elicitor-active oligogalacturonides. Elucidation of regulatory elements of $P G$ and $P G I P$ transcription may provide insights into the complexity of the interaction and the subtleties of the manipulative nature of the parasite. Hence, if $P G$ levels are reduced, the constitutively expressed levels of PGIP should remain unaltered, as observed in incompatible SCN-soybean interactions. After mechanical wounding of roots, levels of $P G$ expression were undetectable, while the $P G I P$ expression was strongly induced in both PI 437654 and Essex. Devoto et al. (1998) reported that the Pgip-1 promoter of Phaseolus vulgaris was induced in response to wounding but not to elicitors or pathogen infection, suggesting multiple members of the $P G I P$ gene family may be differentially regulated depending upon the stimuli. We speculate differential regulation of $P G I P$ transcription to biotic or abiotic stresses and normal developmental processes in soybean roots. Although speculative at this juncture, the transcriptional regulation of endogenous $P G$ could have been exploited by SCN to allow for successful parasitism of soybeans. The cis-acting elements and transacting factors controlling $P G$ transcription may provide some clues for engineering resistance to $\mathrm{SCN}$ in soybeans. 


\section{MATERIALS AND METHODS}

\section{Plant materials.}

Two soybean genotypes, differing in response to SCN, were used in DD analysis. PI 437654 is a plant introduction from China and is resistant to all known races of SCN in the U.S. (Anand 1991; Rao-Arelli et al. 1992). Essex is a commercial cultivar that is susceptible to all races of SCN (Smith and Camper 1973). Nematode populations behaving like race 3 or race 14 according to the race classification scheme (Riggs and Schmitt 1988) were used for infections in our experiments. Three-day-old seedlings of SCN resistant PI 437654 and susceptible Essex were used for inoculations with race 3 as described in Mahalingam et al. (1998). For the analysis of $P G$ and PGIP expression in infected plants, roots were harvested 1, 1.5, 2, 3, and 5 DAI, and leaves and hypocotyls, 2 DAI. Roots, leaves, and hypocotyls of noninfected seedlings were controls. Mechanically wounded roots (harvested $24 \mathrm{~h}$ after piercing with a $18 \mathrm{G}$ needle to simulate nematode penetration) were also analyzed. Five SCN-susceptible soybean genotypes (Brim, Dillon, Hutcheson, Lee, and Perrin) and six soybean genotypes with resistance to at least one $\mathrm{SCN}$ race (Bedford, PI 438489B, PI 209332, PI 90763, PI 88788, and Peking) were included in the expression analysis of $P G$ and $P G I P$. Roots of Peking (resistant to race 3, susceptible to race 14), PI 88788 (resistant to race 3 , moderately resistant to race 14 ), PI 437654, and Essex were infected with races 3 or 14 and were collected 3 DAI.

\section{Isolation of $P G$ by $\mathrm{DD}$.}

Total RNA of PI 437654 and Essex roots, 2 DAI, and noninfected controls were isolated with the RNeasy Plant Isolation Kit (Qiagen, Chatsworth, CA). For every RNA tested, two independent samples were processed. The RNA was treated with RNase-free DNase (Promega, Madison, WI) to remove chromosomal DNA contamination. The reverse transcriptions and PCR analyses were conducted as described in Liang and Pardee (1992). With the combination of T17-C anchor primer and decamer S07: 5'-TCCGATGCTG-3' (Operon Technologies, Alameda, CA), a 350-bp fragment that was differentially expressed in susceptible Essex was identified. This band was reproducibly observed in independent samples and was isolated from the gel, cloned into the pGEM vector (Promega). The cloned fragment was sequenced with the ABI Prism Dye Terminator Cycle Sequencing Ready Reaction Kit (Perkin Elmer, Foster City, CA). Sequencing was conducted in an ABI 131 automated DNA sequencer at Clemson University. Sequences were tested for homology to other sequences with the NCBI's BLAST algorithm (Altschul et al. 1990).

\section{$5^{\prime}$ and $3^{\prime}$ RACE PCR to isolate full-length cDNA of $\boldsymbol{P G}$.}

Based on the sequence data of the isolated DD clone, two primers were synthesized (Integrated DNA Technologies, Coralville, IA) for the $3^{\prime}$ RACE (5'-GCTTGCGGCGATGA TTGCG-3'; 5'-ATGTACACAATGTGAATTGTGG-3') and two for the $5^{\prime}$ RACE (5'-CTGTTACACCATCACTTCC-3'; 5'-ATAGAACCTGAGTGCTGTTGG-3'). Total RNA from PI 437654 and Essex was used in the RACE analysis. The cDNA synthesis and subsequent PCRs were performed according to the kit protocol (GibcoBRL, Rockville, MD). Several clones from the $5^{\prime}$ and $3^{\prime}$ RACE PCR were analyzed and an overlap- ping consensus sequence of 1,676 bp was assembled. Based on this sequence, two primers (5'-ATATGAAACTAAACA CCATTGC-3'; 5'-GTAAATCTTCTCCCCCAACAT-3') were designed to amplify a full-length $P G$ cDNA from Essex and PI 437654. The 1.5-kb fragment was isolated from a lowmelting agarose gel and purified with the Wizard PCR preps (Promega), cloned into pGEM vector (Promega) and sequenced as described above.

\section{Identification of two $P G$ cDNAs in roots.}

Sequencing analysis of more than 20 clones of the $1.5-\mathrm{kb}$ coding $P G$ sequence clearly indicated that there were two $P G$ sequences, designated as $P G 1$ and $P G 2$. CAPS analysis was used to distinguish between $P G 1$ and $P G 2$ cDNAs. Genespecific primers were designed for the two $P G$ cDNAs. For $P G 1$, primers were PG1-1 (5'-GAAAGCTGCTGCATCTGC TACT-3'), PG1-2 (5'-AGAGACGCGCAATCATCGCCG-3'), and PG1-3 (5'-AAGTAGTATAGGCTTGTAAATC-3'). For the $P G 2$ sequence: PG2-1 (5'-CAAAGCTGCCTATGCTAC AACT-3'), PG2-2 (5'-TAGAGATACAGTCATCACCA-3' and PG2-3 (5'-AGCATATGGCTAAGTAAAT-3'). RT-PCR was conducted for 30 cycles at $94^{\circ} \mathrm{C}$ for $40 \mathrm{~s}, 59^{\circ} \mathrm{C}$ for $40 \mathrm{~s}$, and $72^{\circ} \mathrm{C}$ for $2 \mathrm{~min}$. The RT-PCR-generated products were digested with PstI for $3 \mathrm{~h}$ and the digested products were separated on a $2 \%$ agarose gel.

\section{Isolation of $P G I P$ cDNA.}

Three micrograms of total RNA was reverse transcribed with oligo $(\mathrm{dT})_{15}$ primer with $\mathrm{RNase} \mathrm{H}^{-}$reverse transcriptase according to the manufacturer's instructions (Gibco-BRL, Bethesda, MD). Root cDNA and genomic DNA from Essex and PI 437654 were used as templates in PCR. The primers (5'-GAGCTATGCAACCCACAAGA-3'; 5'-TTAAGTGCAG GAAGGAAGAG-3') and PCR amplification conditions were as described in Favaron et al. (1994). The products of PCRs were separated on $1.4 \%$ agarose gel. The amplified fragment (approximately $950 \mathrm{bp}$ ) was excised from the gel and purified with the QIAEX II gel extraction kit (Qiagen), cloned into pGEM vector, and several clones were sequenced as described earlier.

\section{RNA and DNA blot analyses.}

Total RNA from noninfected roots, $\mathrm{SCN}$ race 3-infected roots $(1,1.5,2,3$, and $5 \mathrm{DAI})$, and wounded roots of PI 437654 and Essex were used to investigate the expression of $P G$ and $P G I P$ transcripts. To examine the systemic expression, RNA was extracted from leaves and hypocotyls (noninfected and 2 DAI) from both genotypes. Root RNA from the SCN susceptible (Dillon, Perrin, Brim, Lee, Hutcheson, and Essex) and resistant (Peking, PI 88788, PI 209332, PI 438489B, PI 90763, Bedford, and PI 437654) lines was used to investigate genotypic differences in $P G$ and PGIP expression. RNA from Peking, PI 88788, PI 437654, and Essex, 3 DAI with SCN races 3 and 14 was used to examine race-specific responses of $P G$ and $P G I P$. RNA gel blots and hybridizations were performed as described in Sambrook et al. (1989). The full-length cDNAs of $P G$ (both $P G 1$ and $P G 2$ ) and PGIP were random primed with $\alpha\left[{ }^{32} \mathrm{P}\right] \mathrm{dCTP}$ (Feinberg and Vogelstein 1983) and used as probes in high stringency hybridizations.

Five restriction enzymes (DraI, EcoRI, EcoRV, HindIII, and TaqI) were used for digesting genomic DNA from PI 
437654 and Essex. Full-length cDNAs of $P G 1, P G 2$, and $P G I P$ were used as probes for hybridizations as described in Skorupska et al. (1993).

\section{ACKNOWLEDGMENTS}

We thank Vance Baird and Emerson Shipe for careful review of the manuscript. Technical contribution no. 4490 of South Carolina Agricultural Experimental Station.

\section{LITERATURE CITED}

Altschul, S. F., Gish, W., Miller, W., Myers, E. W., and Lipman, D. J. 1990. Basic local alignment search tool. J. Mol. Biol. 215:403-410.

Anand, S. C. 1991. Registration of soybean germplasm line S88-2036 having multiple race soybean cyst nematode resistance. Crop Sci. $31: 856$.

Bird, C. R., Smith, J. S., Ray, J. A., Moureau, P., Bevan, M. V., Bird, A. S., Hughes, S., Morris, P. C., Grierson, D., and Schuch, W. 1988. The tomato polygalacturonase gene and ripening-specific expression in transgenic plants. Plant Mol. Biol. 11:651-662.

Bird, D. M. 1996. Manipulation of host gene expression by root-knot nematodes. J. Parasitol. 82:881-888.

Brown, S. M., and Crouch, M. L. 1990. Characterization of a gene family abundantly expressed in Oenothera organensis pollen that shows sequence similarity to polygalacturonase. Plant Cell 2:263-274.

Cervone, F., Hahn, M. G., De Lorenzo, G., Darvill, A., and Albersheim, P. 1989. A plant protein converts a fungal pathogenesis factor into an elicitor of plant defense responses. Plant Physiol. 90:542-548.

De Lorenzo, G., Cervone, F., Bellincampi, D., Caprari, C., Clark, A. J., and Desiderio, A. 1994. Polygalacturonase, PGIP and oligogalacturonides in cell-cell communication. Biochem. Soc. Trans. 22:394397.

DellaPenna, D., Alexander, D. C., and Bennett, A. B. 1986. Molecular cloning of tomato fruit polygalacturonase: Analysis of polygalacturonase mRNA levels during ripening. Proc. Natl. Acad. Sci. USA 83: 6420-6424

Desiderio, A., Aracri, B., Leckie, F., Mattei, B., Salvi, G., Tigelaar, H., Van Roekel, J. S. C., Baulcombe, D. C., Melchers, L. S., De Lorenzo, G., and Cervone, F. 1997. Polygalacturonase-inhibiting proteins (PGIPs) with different specificities are expressed in Phaseolus vulgaris. Mol. Plant-Microbe Interact. 10:852-860.

Devoto, A., Leckie, F., Lupotto, E., Cervone F., and De Lorenzo, G. 1998. The promoter of a gene encoding a polygalacturonaseinhibiting protein of Phaseolus vulgaris L. is activated by wounding but not by elicitors or pathogen infection. Planta 205:165-174.

Diers, B. W., Skorupska, H. T., Rao-Arelli, A. P., and Cianzio, S. R. 1997. Genetic relationships among soybean plant introductions with resistance to soybean cyst nematodes. Crop Sci. 37:1966-1972.

English, P. D., Maglothin, A., Keegstra, K., and Albersheim, P. 1972. A cell-wall degrading endopolygalacturonase secreted by Colletotrichum lindemuthianum. Plant Physiol. 49:293-297.

Favaron, F., D’Ovidio, R., Porceddu, E., and Alghisi, P. 1994. Purification and molecular characterization of a soybean polygalacturonaseinhibiting protein. Planta 195:80-87.

Feinberg, A. P., and Vogelstein, B. 1983. A technique for radiolabeling DNA restriction fragments to high specific activity. Anal. Biochem. 132:6-13.

Grundler, F. M., Bockenhoff, W. A., Schmidt, K.-P., Sobczak, M., Golinowski, W., and Wyss, U. 1994. Arabidopsis thaliana and Heterodera schachtii: A versatile model to characterize the interaction between host plants and cyst nematodes. Pages 171-180 in: Advances in Molecular Plant Nematology. F. Lamberti, C. De Georgi, and D. M. Bird, eds. Plenum Press, New York.

Hadfield, K. A., and Bennett, A. B. 1998. Polygalacturonases: Many genes in search of a function. Plant Physiol. 117:337-343.

Hadfield, K. A., Rose, J. K. C., Yaver, D. S., Berka, R. M., and Bennett, A. B. 1998. Polygalacturonase gene expression in ripe melon fruit supports a role for polygalacturonase in ripening-associated pectin disassembly. Plant Physiol. 117:363-373.

Hahn, M. G., Bucheli, P., Cervone, F., Doares, S. H., O’Neill, R. A., Darvill, R. A., and Albersheim, P. 1989. The role of cell wall con- stituents in plant-pathogen interactions. Pages 131-181 in: PlantMicrobe Interactions: Molecular and Genetic Perspectives. T. Kosuge and E. W. Nester, eds. McGraw Hill, New York.

Hahn, M. G., Darvill, A. G., and Albersheim, P. 1981. Host-pathogen interactions XIX. The endogenous elicitor, a fragment of a plant cell wall polysaccharide that elicits phytoalexin accumulation in soybeans. Plant Physiol. 68:1161-1169.

Jones, D. A., and Jones, J. D. G. 1997. The role of leucine-rich repeat proteins in plant defense. Adv. Bot. Res. 24:89-166.

Jones, M. G. K. 1981. Host cell responses to endoparasitic nematode attack: structure and function of giant cells and syncytia. Ann. Appl. Biol. 97:353-372.

Kalatizis, P., Koehler, S. M., and Tucker, M. L. 1995. Cloning of a tomato polygalacturonase expressed in abscission. Plant Mol. Biol. 28: 647-656.

Liang, P., and Pardee, A. 1992. Differential display of eukaryotic messenger RNA by means of polymerase chain reaction. Science 257: 967-971.

Mahalingam, R., Knap, H. T., and Lewis, S. A. 1998. Inoculation method for studying early responses of Glycine max to Heterodera glycines. J. Nematol. 30:237-240.

Mahalingam, R., and Skorupska, H. T. 1996. Cytological expression of early response to infection by Heterodera glycines Ichinohe in resistant PI 437654 soybean. Genome 39:986-998.

Munoz, J. A., Coronado, C., Perez-Hormaeche, J., Kondorosi, A., Ratet, P., and Palomares, A. J. 1998. MsPG3, a Medicago sativa polygalacturonase gene expressed during the alfalfa-Rhizobium meliloti interaction. Proc. Natl. Acad. Sci. USA 95:9687-9692.

Nothangel, E. A., McNeil, M., Albersheim, P., and Dell, A. 1983. Hostpathogen interactions XXII. A galacturonic acid oligosaccharide from plant cell walls elicit phytoalexins. Plant_Physiol. 71:916-926.

Petersen, M., Sander, L., Child, R., Onckelen, H., Ulvskov, P., and Borkhardt, B. 1996. Isolation and characterization of a pod dehiscence zone-specific polygalacturonase from Brassica napus. Plant Mol. Biol. 31:517-527.

Rao-Arelli, A. P., Wrather, J. A., and Anand, S. C. 1992. Genetic diversity among isolates of Heterodera glycines and sources of resistance in soybeans. Plant Dis. 76:894-896.

Riggs, R. D., and Schmitt, D. P. 1988. Complete characterization of the race classification scheme for Heterodera glycines. J. Nematol. 20: 392-395.

Sambrook, J., Fritsch, E. F., and Maniatis, T. A. 1989. Electrophoresis of RNA through gels containing formaldehyde. Pages 7.43-7.45 in: Molecular Cloning: A Laboratory Manual. 2nd ed. J. Sambrook, E. F. Fritsch, and T. A. Maniatis, eds. Cold Spring Laboratory, Cold Spring Harbor, NY.

Skorupska, H. T., Shoemaker, R. C., Warner, A., Shipe, E. R., and Bridges, W. C. 1993. Restriction fragment length polymorphism in soybean germplasm of southern USA. Crop Sci. 33:1169-1176.

Slater, A., Maunders, M. J., Edwards, K., Schuch, W., and Grierson, D. 1985. Isolation and characterization of cDNA clones for tomato polygalacturonase and other ripening-related proteins. Plant Mol. Biol. 5:137-147

Smith, T. J., and Camper, H. M. 1973. Registration of Essex soybean. Crop Sci. 13:495

Stotz, H. U., Powell, A. L. T., Damon, S. E., Greve, C. G., Bennett, A. B., and Labavitch, J. M. 1993. Molecular characterization of a polygalacturonase inhibitor from Pyrus communis L. cv. Bartlett. Plant Physiol. 102:133-138.

Taylor. J. E., Webb, S. T. J., Coupe, S. A., Tucker, G. A., and Roberts, J. A. 1990. Changes in polygalacturonase activity and solubility of polyuronides during ethylene-stimulated leaf abscission in Sambucus nigra. J. Exp. Bot. 44:93-98.

Tebutt, S. J., Rogers, H. J., and Lonsdale, D. M. 1994. Characterization of a tobacco gene encoding a pollen-specific polygalacturonase. Plant Mol. Biol. 25:283-297.

Walker-Simmons, M., Jin, D., West, C. A., Hadwiger, L., and Ryan, C. A. 1984. Comparison of proteinase-inhibitor-inducing activities and phytoalexin elicitor activities of a pure fungal endopolygalacturonase, pectic fragments, and chitosans. Plant Physiol. 76:833-836.

Wing, R. A., Yamaguchi, J., Larabell, S. K., Ursin, V. M., and McCormick, S. 1989. Molecular and genetic characterization of two pollenexpressed genes that have sequence similarity to pectate lyases of the plant pathogen Erwinia. Plant Mol. Biol. 14:17-28. 